\title{
MULTIMODAL IMPACT OF ACUPUNCTURE, EXERCISE THERAPY, AND CONCURRENT FUNCTIONAL ELECTRICAL STIMULATION ON OSTEOARTHRITIS OF THE KNEE: A CASE REPORT
}

\author{
Azadeh Hakakzadeh, MD \\ Sports Medicine Research Center, Neuroscience Institute, Tehran University of Medical \\ Sciences, Tehran, Iran \\ Ardalan Shariat, PhD \\ Sports Medicine Research Center, Neuroscience Institute, Tehran University of Medical \\ Sciences, Tehran, Iran \\ Vahide Moradi, PhD \\ Dept. of Orthotics and Prosthetics, Faculty of Rehabilitation, Iran University of Medical \\ Sciences, Tehran, Iran \\ Lee Ingle, PhD \\ Dept. of Sport, Health \& Exercise Science, University of Hull, Kingston-upon-Hull, United \\ Kingdom \\ Roshanak Honarpishe, PhD \\ Dept. of Physiotherapy, School of Rehabilitation, Tehran University of Medical Sciences, \\ Tehran, Iran

\section{Pardis Noormohammadpour, MD} \\ Sports Medicine Research Center, Neuroscience Institute, Tehran University of Medical \\ Sciences, Tehran, Iran

\section{Noureddin Nakhostin Ansari, PhD} \\ Dept. of Physiotherapy, School of Rehabilitation, Tehran University of Medical Sciences, \\ Tehran, Iran
}

Running head: Multidisciplinary treatment for knee pain

Declaration of Conflicting Interests: The author(s) declared no potential conflicts of interests with respect to the research, authorship, and/or publication of this article.

Funding: There was no funding for this research. 
*Corresponding Author: Ardalan Shariat, Sports Medicine Research Center, Neuroscience Institute, Tehran University of Medical Sciences, Tehran, Iran. E-mail:

ardalansh2002@gmail.com.

Authors' accepted manuscript of article:

Hakakzadeh, Azadeh, et al. "Multimodal Impact of Acupuncture, Exercise Therapy, And Concurrent Functional Electrical Stimulation On Osteoarthritis of the Knee: a Case Report." Acupuncture \& ElectroTherapeutics Research, vol. 44, no. 2, 2019, pp. 135-145., doi:10.3727/036012919x15650315071942.

\section{ABSTRACT:}

Knee osteoarthritis (OA) causes functional limitation in weight-bearing activities including walking. To investigate the multimodal impact of acupuncture, exercise therapy, and concurrent functional electrical stimulation (FES) on knee osteoarthritis. We designed a multidisciplinary treatment package including acupuncture; home based exercise therapy, and concurrent functional electrical stimulation during treadmill walking. Outcomes measurements included the numerical rating scale (NRS), the Knee Injury and Osteoarthritis Outcome Score (KOOS), and the Tampa Scale of Kinesiophobia (TSK). Measurements were completed at baseline and following the treatment phase which consisted of six individual sessions. A 48-year-old male, office worker presented with a history of chronic right knee. During the previous year, he was diagnosed with knee osteoarthritis after clinical physical examination by a sports medicine physician. Following our novel training intervention, the patient reported a reduction in pain intensity from 8 to 2 on the NRS, improved in all KOOS subscale scores, and improved in the TSK scale (reduction from 15 to 11). In addition, the patient reported that he was able to return to work and undertake normal activities of daily living with reduced knee pain. This case report showed that our novel multimodal intervention including six sessions of acupuncture, exercise therapy, and treadmill walking with 
functional electrical stimulation (FES) had a positive impact on knee pain and function in a middleaged male with knee osteoarthritis.

Keywords: exercise, acupuncture, chronic knee osteoarthritis, combined therapy

\section{Introduction}

Osteoarthritis $(\mathrm{OA})$ is the most common cause of chronic knee pain, affecting millions of people worldwide [1]. It occurs due to a degeneration of protective cartilage at the bone-ends over time. Although osteoarthritis can impact any joint in the body, it commonly affects joints in the hands, knees, hips, and spine [1, 2], however, prevalence is greatest in the hips and knees [3]. Numerous management strategies exist for the treatment of knee OA [4]. Current therapeutic approaches include non-pharmacological and pharmacological methods [5]. Pharmacological approaches are generally limited to the use of acetaminophen or non-steroidal anti-inflammatory agents (NSAIDs) [5], despite the use of these drugs, the majority of patients report persistent pain.[5] Physical therapy [5], exercise [6], weight loss [2], acupuncture [7] and methods of unloading joints including the use of canes and walkers [5] are frequently used as initial approaches to therapy, or as adjuncts to pharmacological approaches [4].

Acupuncture is one of the non-pharmacological treatment techniques used to manage chronic pain in patients with OA of the knee [8]. Few robust, well designed studies exist, through early indications would suggest that acupuncture appears to be effective for reducing pain and improving function in patients $[9,10]$. A recent meta-analysis showed that acupuncture can contribute to shorter- and long-term improvements in knee function in patients with chronic knee pain due to osteoarthritis [7]. Functional Electrical Stimulation (FES) is a rehabilitation device used to facilitate muscular action [11]. The FES device produces a skeletal muscle contraction through the percutaneous stimulation of peripheral nerves, aiming to preserve 
muscle mass and strength and prevent muscle atrophy $[12,13]$. It has been used in combination with gait training for patients following stroke, and in patients with multiple sclerosis and Parkinson's disease to improve gait function $[14,15]$. We have developed a unique multimodal approach to chronic knee pain which combines exercise therapy, acupuncture, and concurrent FES during treadmill walking. This case report describes the outcomes of this approach following a short-term (6 sessions) intervention on knee pain and function in a middle-aged male patient suffering from OA in the right knee. To our knowledge, there are no published reports that have combined these therapies into one multimodal treatment battery.

\section{Materials and methods}

\section{Case history}

Study approval was obtained from the Research Ethics Committee of the Sports Medicine Research Center, Tehran. A 48-year-old male office worker who consistently stood for around 8 hours per day during working days, was admitted to the sports medicine research center, neuroscience institute. His stature was $1.79 \mathrm{~m}$, and his body mass was $111 \mathrm{~kg}$ (body mass index of $34.6 \mathrm{~kg} \cdot \mathrm{m}^{-2}$ ). The patient had a history of right knee pain for approximately 1 year. His pain worsened while standing for long periods. He complained of muscle cramps in his right quadriceps for the past 3 months. One year ago, he was admitted to an emergency department because of an acute right knee joint effusion. The effusion was aspirated and, at that point, he underwent knee arthrocentesis due to suspicion of septic knee arthritis. However, subsequent fluid culture ruled out sepsis. The rheumatologist also undertook a pulmonary CT scan, blood, and urinary analysis, at which point he was admitted to hospital for one month. None of the investigations revealed a 
specific cause. At this time, the patient failed to undertake any rehabilitative exercise during the admission. His knee pain re-started after discharge from hospital.

\section{Evaluation}

In his first visit to the clinic, the physical examination indicated that the patient had no joint effusion, had full active range of motion in the right knee, and right medial joint line tenderness in palpation. The patella was laterally tilted, valgus and varus stress test was normal, the McMurray test was negative, anterior and posterior drawer test was normal. He was diagnosed with knee osteoarthritis according to American College of Rheumatology (ACR) Clinical Classification Criteria for Osteoarthritis of the Knee [16]. His osteoarthritis was Grade 1 based on Kellgren Lawrence grading (Fig. 1) [17].

We assessed his pain and function by two questionnaires. The Knee Injury and Osteoarthritis Outcome Score (KOOS), has been shown to have acceptable levels of reliability and validity [18]. The KOOS consists of 5 subscales; pain, other symptoms, function in daily living (ADL), function in sport and recreation (Sport/Rec) and knee related Quality of Life (QoL). The Tampa Scale of Kinesiophobia (TSK) was also utilised as it was developed for quantifying fear of injury especially in patient with knee injuries [19]. This questionnaire includes 17 statements in which each question is provided with four-point Likert scale ranging from 0 (strongly disagree) to 3 (strongly agree). The total score that can be achieved is 51 , with higher scores associated with greater fear. It is noteworthy, 4 items $(4,8,12$, and 16) were negatively worded and reverse scored. Previous studies have demonstrated the validity and reliability of TSK in patients with chronic musculoskeletal disorders $[20,21]$. We asked the patient to rate his pain on a 0 to 10 scale. The NRS has been shown to have acceptable levels of reliability and validity [22]. 

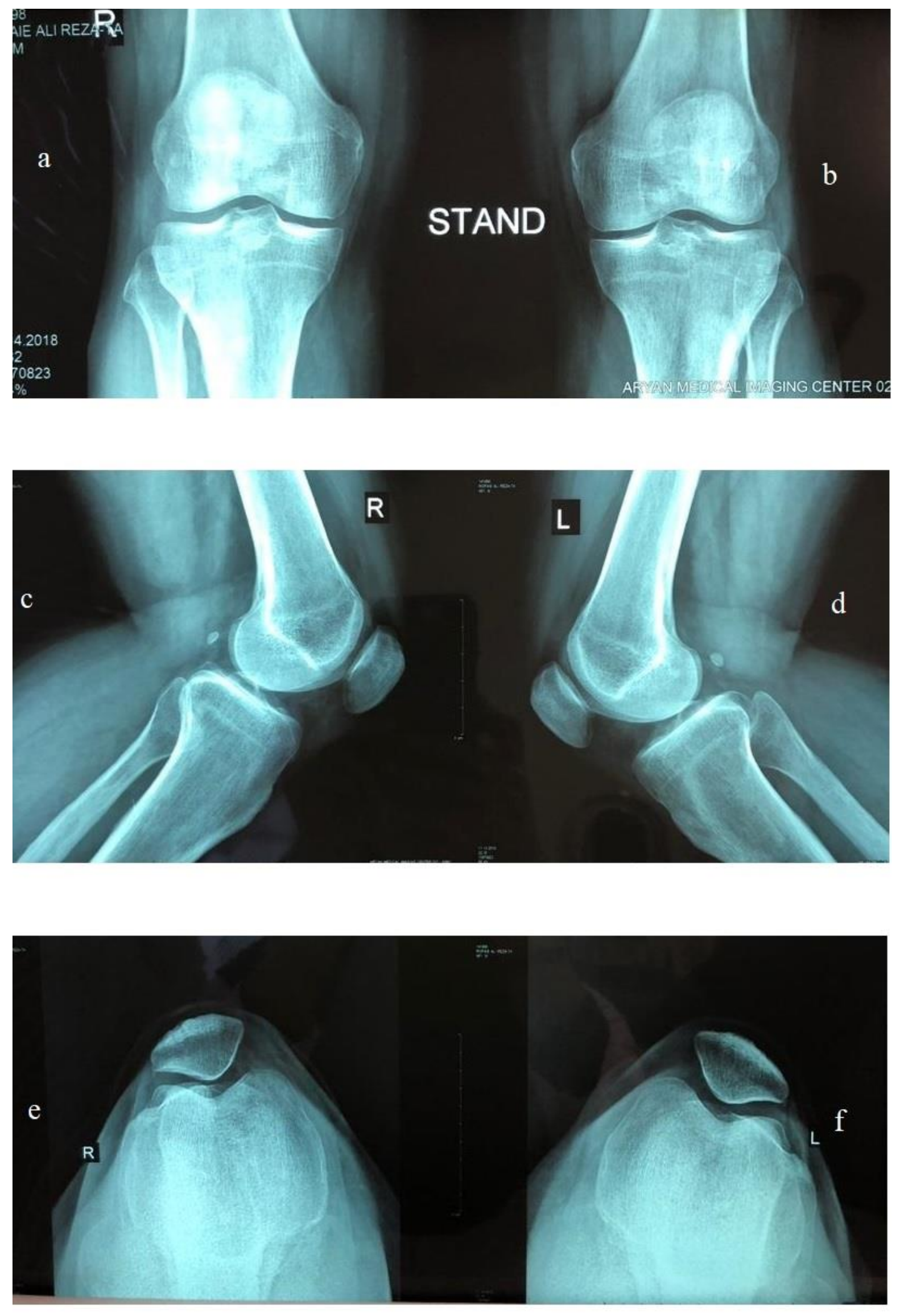


\section{Figure 1.}

a. Anteroposterior standing radiograph of knee joint (right side)

b. Anteroposterior standing radiograph of knee joint (left side)

c. Lateral view of knee joint radiography (right side)

d. Lateral view of knee joint radiography (left side)

e. Sunrise view of knee radiography (right side)

f. Sunrise view of knee radiography (left side)

\section{Multimodal intervention}

We designed a specific treatment package including acupuncture (Fig. 2), concurrent functional electrical stimulation (RehaMove, Hasomed, Germany) with treadmill (TechnoGym, Italy) walking (Fig.3), supervised strengthening exercises, and home based isometric exercises for six sessions (Table 1). The first therapy session was performed in the clinic, and we asked the patient to perform the same exercises at home every day. Based on current evidence [23-26], apuncture was directed on acupoints of the stomach (ST36) or zu san li, ST33, ST 34, ST 35, spleen (SP9), SP10, extra lower extremity (Ex-LE 4), (Ex- LE2), and gallbladder (GB) 34 in the first session, and we also checked the patient for quadriceps trigger points and existence of pes anserine tenderness. We applied needles on pes anserine tender points and quadriceps trigger points. (Fig. 4) [27]. The number of needles in the first session was based on the trigger points and the location of pain. During the treatment sessions, the numbers of needles on the quadriceps trigger points (4 needles), and pes anserine tender points (4 needles), were decreased, so, by the third session of treatment, there was no trigger point, and we only applied six needles on the aforementioned accupoints . An experienced sports medicine specialist, with cooperation of an experienced physiotherapist and neurorehabilitation specialist was responsible for the needle application. 
Table 1. Package of treatment

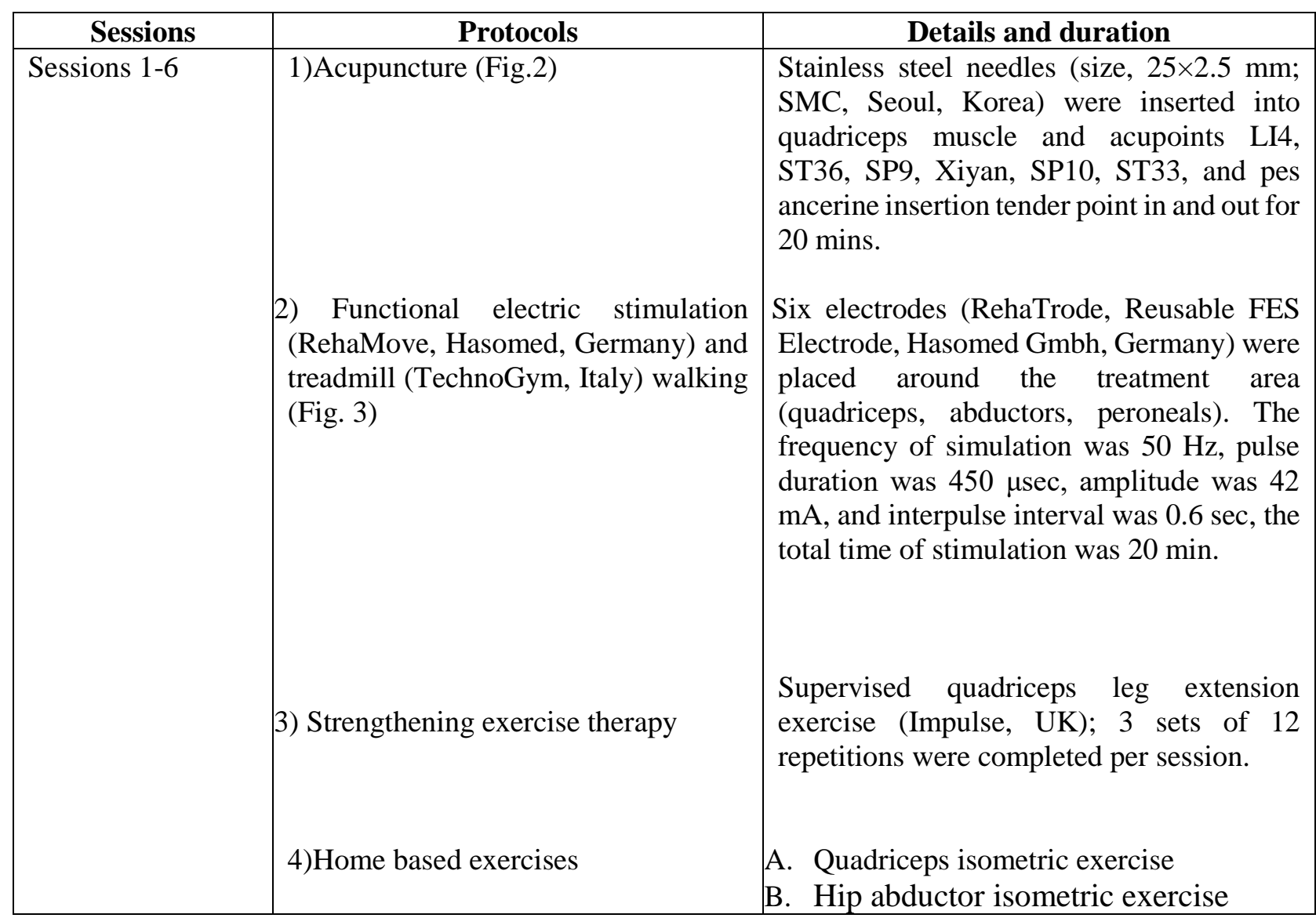




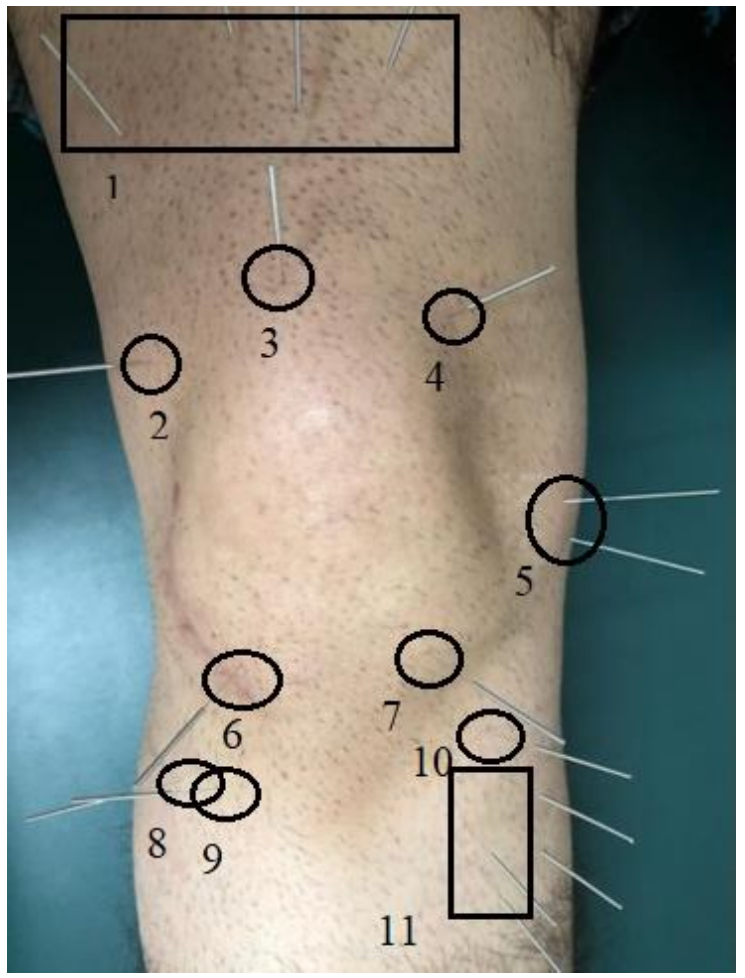

Fig.2 Location of acupuncture points: 1. Quadriceps trigger points (4 needles), 2. ST-34 (1 needle), 3.ExLE 2 (1 needle), 4. Ex-LE 3 (1 needle), 5.Medial knee joint line tender points ( 2 needles), 6.ST-35 (1 needle), 7.Ex-LE 4 (1 needle), 8. GB-34 (1 needle), 9. ST-36 (1 needle), 10.SP-9 (1 needle), 11. Pes anserine tender points (4 needles). 


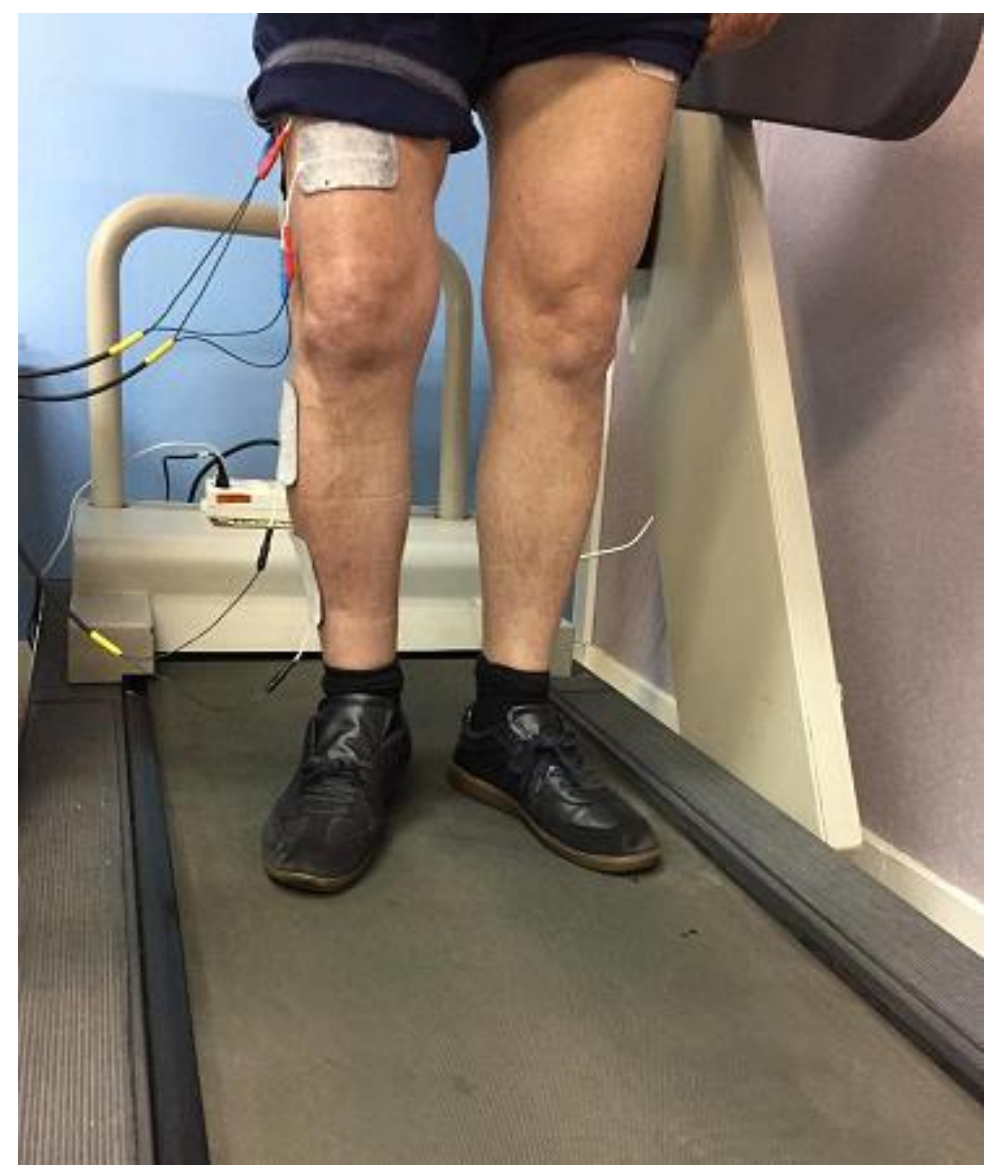

Fig 3. Functional electrical stimulation and treadmill walking.

\section{Results}

At baseline, the KOOS pain score was 72, symptoms 82, KOOS ADL 68, KOOS Sport/Rec was 20 and KOOS QOL was 44 (Table 2) [28]. TSK score was 15 before treatment. The pain severity was scored " 8 " based on the NRS. All measurements were repeated after the sixth (and final) session of intervention. The patient reported much less pain during walking and the NRS pain scale fell by six points to 2 points after the intervention. His KOOS pain score was 89 , symptoms 86 , KOOS ADL 82, KOOS Sport/Rec was 80 and KOOS QOL was 50. Our patient improved in all 
subscales of KOOS following our treatment package (Table 2). Finally, the TSK was reduced from 15 to 11 . In addition, the intervention also led to a reduction in fear of re-injury (Table 2).

Table 2. Pain intensity and questionnaire sub-scales before and after the intervention

\begin{tabular}{|l|l|l|}
\hline \multicolumn{1}{|c|}{ Variable } & \multicolumn{1}{|c|}{ Pre-intervention } & Post-intervention \\
\hline Numeric rating scale & 8 & 2 \\
KOOS subscales & 72 & 89 \\
Pain & 68 & 82 \\
Symptoms & 20 & 80 \\
ADL & 68 & 82 \\
Sport/Rec & 44 & 50 \\
QOL & & \\
TSK & 15 & 11 \\
\hline
\end{tabular}

\section{Discussion}

There is little agreement regarding the optimal therapy for pain reduction in patients with knee OA $[5,29]$. Our case report showed that a multimodal treatment package including acupuncture, exercise therapy, and FES during walking produced a significant reduction in knee pain and function after six therapy sessions. In our view, it is difficult to distinguish which of the individual approaches was the most effective. Our study tested each approach in combination, therefore, we could only speculate which one was the most effective. Further research studies with differing designs, comparing techniques in isolation and in combination would be required to answer this question.

Acupuncture is used to improve pain and function as a treatment technique to manage knee OA [30]. A systematic review reported positive effects on knee pain and emphasized its safety in comparison to standard pharmacological therapy [31]. 
Studies have shown that exercise therapy results in the reduction of knee pain, although no general consensus exists regarding the most efficacious approach [29]. The effectiveness of exercise programs within clinical trials are variable and are difficult to compare; the interventions differ substantially in terms of type of exercise (aerobic, strengthening, etc.), intensity of exercise, duration of intervention, and number of sessions per week [32]. Furthermore, the patients included are often heterogeneous in age, sex, body mass index (BMI), radiographic severity of OA [32]. In our intervention, we used a combination of isometric (for home-based exercise) and isotonic (for facility-based exercise), in addition, treadmill walking was also included.

Rosemffet et al. reported that therapeutic electrical stimulation applied in combination with exercise was a useful therapeutic alternative for patients with OA of the knee [33]. However, a recent systematic review revealed that neuromuscular electrical stimulation had no benefits on pain reduction [34]. Matsuse et al. included walking exercise simultaneously combined with neuromuscular electrical stimulation of the quadriceps and hamstring for 12 weeks; they reported improvement in muscle strength, physical function, and knee pain in symptomatic knee osteoarthritis [35]. In our case study, gait training with FES lead to a significant improvement after only six sessions [36]. Different treatment modalities including foot bracing and quadriceps strengthening has previously been shown to improve the walking ability [37]. Combined therapy could improve coordination between different muscles and help facilitate the co-ordination of complex movements [38]. In patients with knee OA, walking is associated with an increased mechanical loading of the ankle, knee and hip joints; mostly in frontal plane [37]. Hence patients with knee OA may require specific hip abductor moments for controlling pelvic level unless they cannot control the medial compartment load of the knee, increasing the risk of OA progression 
[37]; Moreover, maximum inversion moments of the ankle, during the stance phase have been reported. Since this abnormal increase may produce a greater split in the knee cartilage, a treatment which focuses on these two compartment muscles seems to be effective [37]. In addition, the importance of quadriceps strength cannot be underestimated in patients with OA of the knee. Quadriceps weakness is a well-established risk factor of knee pain and is associated with balance and postural sway deficiency [39]. We acknowledge that other studies have emphasised the longer term benefits of low frequency stimulation, and this may be more effective than high frequency stimulation for longer lasting pain relief $[40,41]$.

\section{Conclusion}

In conclusion, our short-term (6 sessions) multimodal treatment package led to a significant improvement in knee pain and function in our patient. However, these results, should be interpreted with caution, as our findings are based on a sample size of one. Future studies focused on robust experimental design should be developed in order to strengthen the existing evidence-base.

\section{References}


1. Vina ER, Kwoh CK. "Epidemiology of osteoarthritis: Literature update". Current Opinion in Rheumatology. Vol.30, pp.160-167, 2018.

2. Plotnikoff R, Karunamuni N, Lytvyak E, Penfold C, Schopflocher D, Imayama I, Johnson ST, Raine K. "Osteoarthritis prevalence and modifiable factors: A population study Chronic Disease epidemiology". BMC Public Health. Vol.15, pp.1-10, 2015

3. Allen KD, Golightly YM, Hill C, Hill C, Hill C, Hill C. "Epidemiology of osteoarthritis: state of the evidence". Departemen of health and human services USA. Vol.27,pp.276283, 2015.

4. McAlindon TE, Bannuru RR, Sullivan MC, et al. "OARSI guidelines for the non-surgical management of knee osteoarthritis". Osteoarthritis and Cartilage. Vol.22, pp.363-388, 2014.

5. Kalunian KC. "Current advances in therapies for osteoarthritis". Current Opinion in Rheumatology. Vol.28, pp.246-250, 2016.

6. Javadian Y, Adabi M, Heidari B, Babaei M, Firouzjahi A, Ghahhari BY, Hajian-Tilaki K. "Quadriceps muscle strength correlates with serum Vitamin D and knee pain in knee osteoarthritis". Clinical Journal of Pain. Vol.33, pp.s67-70, 2017.

7. Xianfeng Lin, Kangmao Huang, Guiqi Zhu, Zhaobo Huang, An Qin, and Shunwu Fan M. "The Effects of Acupuncture on Chronic Knee Pain". Journal of Bone and Joint Surgery. Vol.98, pp. 1578-1585, 2016.

8. Birch S, Lee MS, Robinson N, Alraek T. The U . K . "NICE 2014 Guidelines for Osteoarthritis of the Knee : Lessons Learned in a Narrative Review Addressing Inadvertent Limitations and Bias". The Journal of Alternative and Complementary Medicine Vol.23, pp.242-246, 2017.

9. Pecos-mart D, Ochoa-s V. "Effects of dry needling in an exercise program for older adults with knee osteoarthritis". Medicine. Vol. 97, pp.43-48, 2018

10. Mayoral O, Salvat I, Martín MT, Martín S, Santiago J, Cotarelo J, Rodríguez C. "Efficacy of Myofascial Trigger Point Dry Needling in the Prevention of Pain after Total Knee Arthroplasty : A Randomized, Double-Blinded, Placebo-Controlled Trial". EvidenceBased Complementary and Alternative Medicine. pp.17-19, 2013.

11. Cienfuegos PJ, Shoemaker A, Grange RW, Abaid N, Leonessa A. "Classical and adaptive control of ex vivo skeletal muscle contractions using functional electrical stimulation (FES)". PLoS ONE. Vol.12, pp.1-29, 2017

12. Shariat A, Ansari NN, Shaw BS. "Cycling training and functional electrical stimulation for post-stroke patients". Revista Brasileira de Medicina do Esporte. Vol.24, pp.300-302, 2018.

13. Bauer P, Krewer C, Golaszewski S, Koenig E, Müller F. "Functional electrical stimulation-assisted active cycling - Therapeutic effects in patients with hemiparesis from 7 days to 6 months after stroke: A randomized controlled pilot study". Archives of Physical Medicine and Rehabilitation. Vol.96, pp.188-196, 2015.

14. Lindquist ARR, Prado CL, Barros RML, Mattioli R, Da Costa PHL, Salvini TF. "Gait training combining partial body-weight support, a treadmill, and functional electrical stimulation: effects on poststroke gait". Physical therapy. Vol.87, pp.1144-1154, 2007.

15. Giesser B, Beres-Jones J, Budovitch A, Herlihy E, Harkema S. "Locomotor training using body weight support on a treadmill improves mobility in persons with multiple sclerosis: a pilot study". Multiple Sclerosis Journal. Vol.13, pp.224-231, 2007.

16. Altman R, Asch E, Bloch D, et al. "Development of criteria for the classification and 
reporting of osteoarthritis classification of osteoarthritis of the knee". Arthritis \& Rheumatism. Vol.29, pp.1039-1049, 1986.

17. Brandt KD, Fife RS, Braunstein EM, Katz B. "Brief reprt radiographic grading of the severity of knee osteoarthritis : relation of the KELLGREN AND LAWRENCE grade to a grade based on joint space narrowing, and correlation with Arthroscopic Evidence of Articular Cartilage Degeneration". Arthritis \& Rheumatism. Vol.34, pp.1381-1386, 1991.

18. Roos EM, Lohmander LS. "The Knee injury and Osteoarthritis Outcome Score ( KOOS ): from joint injury to osteoarthritis Pilot study". Health and quality of life outcomes. Vol.8, pp.1-8, 2003.

19. Kori SH. "Kinisophobia: a new view of chronic pain behavior". Pain Manage. 35-43, 1990.

20. French DJ, France CR, Vigneau F, French JA, Evans RT. "Fear of movement/(re) injury in chronic pain: a psychometric assessment of the original English version of the Tampa scale for kinesiophobia (TSK)". Pain. Vol.127, pp.42-51, 2007.

21. Lundberg MKE, Styf J, Carlsson SG. "A psychometric evaluation of the Tampa Scale for Kinesiophobia-from a physiotherapeutic perspective". Physiotherapy theory and practice. Vol.20, pp.121-133, 2004.

22. Welsh JT. "Assessing pain in the ED including the use of pain scales (such as OSBD, FLACC, VRS, NRS, CRS, and Oucher)". Current Emergency and Hospital Medicine Reports. Vol. 4, pp.19-25, 2016.

23. Ulett, G. A., Han, J., \& Han, S. "Traditional and evidence-based acupuncture". Southern Medical Journal. Vol.91, pp. 1115-1120. 1998.

24. Barlas P. "An introduction to western medical acupuncture". Focus On Alternative And Complementary Therapies An Evidence-based Approach. Vol. 14, pp152-157, 2009.

25. Berman, B. M., Swyers, J. P., \& Ezzo, J. "The evidence for acupuncture as a treatment for rheumatologic conditions". Rheumatic Disease Clinics of North America, Vol. 26, 103 $115,2000$.

26. White A, Tough L, Eyre V, Vickery J, Asprey A, Quinn C, Warren F, Pritchard C, Foster NE, Taylor RS." Western medical acupuncture in a group setting for knee osteoarthritis: results of a pilot randomised controlled trial". Pilot and feasibility studies. Vol 2, pp.1012.

27. M- BSS. "Acupuncture of the Knee". pp, 56-74, 1991.

28. Koos T, Living D, Function R. "Knee injury and Osteoarthritis Outcome Score". pp.1-5, 2012.

29. Juhl C, Christensen R, Roos EM, Zhang W, Lund H. "Impact of Exercise Type and Dose on Pain and Disability in Knee Osteoarthritis A Systematic Review and Meta-Regression Analysis of Randomized Controlled Trials". Vol.66, pp.622-636, 2014.

30. Yang M, Jiang L, Wang Q, Chen H, Xu G. "Traditional Chinese medicine for knee osteoarthritis: An overview of systematic review". PloS one. 12:e0189884, 2017.

31. Kwon YD, Pittler MH, Ernst E. "Acupuncture for peripheral joint osteoarthritis: a systematic review and meta-analysis". Rheumatology. Vol. 45, pp.1331-1337, 2006.

32. Fransen M, Mcconnell S, Harmer AR, Esch M Van Der, Simic M, Bennell KL. "Exercise for osteoarthritis of the knee : a Cochrane systematic review". British Journal of Sports Medicine. Vol. 49, pp.1554-1557, 2015.

33. Rosemffet MG, Schneeberger EE, Citera G, Sgobba ME, Laiz C, Schmulevich H, Artçanuturry P, Gagliardi S, Maldonado Cocco JA. "Effects of functional 
electrostimulation on pain, muscular strength, and functional capacity in patients with osteoarthritis of the knee". Journal of Clinical Rheumatology. Vol.10, pp.246-249, 2004.

34. Zeng C, Yang T, Deng Z, Yang Y, Zhang Y, Lei G. "Electrical stimulation for pain relief in knee osteoarthritis: systematic review and network meta-analysis". Osteoarthritis and cartilage. Vol.23, pp.189-20, 2015.

35. Matsuse H, Hashida R, Takano Y, Omoto M, Nago T, Bekki M, Shiba N. "Walking exercise simultaneously combined with neuromuscular electrical stimulation of antagonists resistance improved muscle strength, physical function, and knee pain in symptomatic knee osteoarthritis: a single-arm study". The Journal of Strength \& Conditioning Research. Vol. 31, pp.171-180, 2017.

36. Rane L, Bull AMJ. "Functional electrical stimulation of gluteus medius reduces the medial joint reaction force of the knee during level walking". Arthritis research \& therapy. Vol. 18, pp.255-259. 2016.

37. Mündermann A, Dyrby CO, Andriacchi TP. "Secondary gait changes in patients with medial compartment knee osteoarthritis: increased load at the ankle, knee, and hip during walking". Arthritis \& Rheumatism. Vol.52, pp.2835-2844, 2005.

38. Paillard T. "Combined application of neuromuscular electrical stimulation and voluntary muscular contractions". Sports medicine. Vol 38, pp.161-177, 2008.

39. Fitzgerald GK, Piva SR, Irrgang JJ, Bouzubar F, Starz TW. "Quadriceps activation failure as a moderator of the relationship between quadriceps strength and physical function in individuals with knee osteoarthritis". Arthritis Care \& Research. Vol. 51, pp.40-48, 2004

40. Omura, Y. "Patho-physiology of acupuncture treatment: effects of acupuncture on cardiovascular and nervous systems". Acupuncture \& Electro-Therapeutics Research. Vol.1,pp. 51-141, 1975

41. Omura, Y. "Electro-acupuncture: its electro-physiological basis and criteria for effectiveness and safety-Part I". Acupuncture \& Electro-Therapeutics Research. Vol 1, pp. 157-181, 1975 